REVIEW

\title{
Treatment of spondylolysis with external electrical stimulation in young athletes: a critical literature review
}

\section{Stasinopoulos}

Br J Sports Med 2004;38:352-354. doi: 10.1136/bjsm.2003.010405

Lumbar spondylolysis is a common cause of low back pain in adolescent athletes. It is a unilateral or bilateral defect of the pars interarticularis. The cause is still a matter of debate. A wide range of conservative treatments has been used. The purpose of this critical literature review is to investigate the efficacy of external electrical stimulation in the healing of this disorder.

$\mathrm{S}$ pondylolysis is a unilateral or bilateral defect of the isthmic portion of the pars interarticularis of a vertebra, without forward displacement of that vertebra on the adjacent vertebra. ${ }^{1-3}$ It is a stress or fatigue fracture seen most often in children and adolescents. ${ }^{2-4}$

It is the most common overuse sporting injury of the lower back, which has been reported to range from $13 \%$ to $47 \%$ among adolescent athletes. $^{5-7}$

The L5 and occasionally the L4 vertebrae are usually involved. ${ }^{3}$ Although spondylolysis has been reported to be more common in male than female patients, progression is more likely in female patients. ${ }^{1}$ The fracture usually occurs on the side opposite to the one performing the activity-that is, left sided fracture in right handed players. ${ }^{9}$

The exact cause of spondylolysis is unclear. It has been described as hereditary, associated with an inherited predisposition to a hypoplastic pars interarticularis, ${ }^{510-12}$ or acquired as the result of repetitive stress and fatigue of the lower segment, leading to a stress reaction and subsequent failure. ${ }^{13}$ The latter hypothesis has led to the postulate that lumbar lordosis, such as is seen in Scheurmann's kyphosis, and sports that demand repetitive hyperextension and rotation of the lumbar spine, such as soccer, wrestling, volleyball, gymnastics, football, and weightlifting, are associated with higher incidences of spondylolysis. ${ }^{9}$ Spina bifida occulta has been noted in patients with spondylolysis and may lead to instability of the lower lumbar segment, predisposing to the development of pars interarticularis defects. ${ }^{14}{ }^{15}$ A relation between spondylolysis and spondylolisthesis has been shown by Ikata et al. ${ }^{5}$ Spondylolysis is a risk factor for the progression to spondylolisthesis.

Many patients with spondylolysis are asymptomatic and therefore require no treatment, but athletes whose sports involve repetitive hyperextension often present initially with pain during certain performance activities. ${ }^{19}$ This pain is either unilateral or bilateral, but it may become a more chronic, dull, midline lumbosacral pain in time. The treatment goals should include pain relief, healing of the spondylolysis, and prevention of further lumbar segment injury.

Studies have shown that patients with spondylolysis may be successfully treated conservatively, but it is not clear from these studies which treatment is the most effective. A wide range of conservative interventions has been advocated. Textbooks of sports medicine, family practice, orthopaedics, and physiotherapy were searched for the management of this condition. The books range over a 15 year span. Medline was searched from 1980 to 2003 in the English language using the following subject terms: spondylolysis and non-operative treatment. The conservative interventions include restriction of activity, ${ }^{16}{ }^{17}$ antilordotic bracing ${ }^{18}$ or bracing that maintains lumbar lordosis, ${ }^{8}$ abdominal strengthening exercises, hamstring stretching, pelvic tilts, ${ }^{8}{ }^{19}$ specific stabilising exercises of muscles surrounding the spine considered to provide dynamic stability and fine control to the lumbar spine, ${ }^{20}$ and last but not least external electrical stimulation. ${ }^{21} 22$ The last conservative measure has not been mentioned in books but was found in two articles, which were written in the last decade. Therefore I decided to focus on the two latter studies, looking particularly at the role of external electrical stimulation in the management of patients with a diagnosis of spondylolysis, as this is not mentioned in books and is something "new" not only to me but also to most physiotherapists.

The purpose of this critical literature review is to establish whether external electrical stimulation can decrease pain and heal the defect of the pars interarticularis, so that this method can be used for the treatment of spondylolysis as the first choice or when other traditional methods have failed and the only solution seems to be surgery.

\section{REVIEW}

Two articles were found in which external electrical stimulation was used to treat patients with spondylolysis. ${ }^{21} 22$ Pettine $e t a^{21}$ presented a case report of a 17 year old male student athlete who had spondylolysis at L4. The initial assessment showed that lumbar extension reproduced the low back pain, without evidence of hamstring tightness and radicular symptoms. Oblique roentgenographs confirmed the diagnosis, and computed tomography (CT) showed

Abbreviations: CT, computed tomography; TLSO, thoracolumbar sacral orthosis 
bilateral pars fractures at L4. The treatment started in the middle of January 1989, with restriction of activity and a thoracolumbar sacral orthosis (TLSO) worn during daily activities. In late February, an external bone growth stimulator was added to the above treatment. The patient was instructed to use it daily, while sleeping. In March 1989 his symptoms were minimal, but a CT scan showed no evidence of healing compared with the first CT scan. In May 1989, a CT scan showed healing of the right pars fracture and progressive healing of the left pars pars fracture. In June 1989, he was pain free and wore the TLSO only during athletic activities. He continued to use the external electrical stimulator daily, while sleeping, until November. A CT scan obtained on 22 November 1989 showed complete healing of the right pars fracture and partial healing of the left pars fracture. One year later, after the same treatment (external electrical stimulation and TLSO), a CT scan showed complete healing of the right pars fracture and solid healing of the left pars fracture.

Background information about the subject's health and how the condition was diagnosed was presented well, but, in the initial assessment, when the pain was reproduced by lumbar extension, no outcome measure (visual analogue scale or pain questionnaire) was used to evaluate the patient's level of pain. Moreover, when the patient had minimal pain and when he was pain free, the authors did not mention how they tried to reproduce the pain and how the level of pain was evaluated. Furthermore, they did not mention how the flexibility of the hamstrings was evaluated. On the other hand, the progressive osseous healing was well presented by the use of CT scans. However, the authors did not report what kind of external bone growth stimulator was used, the frequency, or for how long the patient used it. They only stated "he was instructed to use it while sleeping". He probably did not sleep for the same number of hours everyday, and even if he did, the authors did not refer to it. In addition, they did not mention whether the procedure was explained to the patient or if his parents gave permission for his participation, because this kind of treatment had never been used in the past. Furthermore, they did not justify why they chose specific dates for reassessment or why the use of external electrical stimulation was suggested one month after the use of bracing. Although three months after starting external electrical stimulation and four months after using TLSO and restriction of activities, a CT scan showed progressive osseous healing, in the discussion section they could not establish with certainty how each of the above three elements of the treatment contributed to the overall results. Also, when the CT scan showed complete healing of the right and left pars fracture, the authors did not mention if the healing of the pars defects occurred as a result of external electrical stimulation or bracing. The method in this case report has major flaws, and it is difficult to replicate the procedure without having to ask for clarification of several points. In the discussion section, the authors recapped the key points of the procedure, discussed the results briefly without reaching any conclusion, and recommended future studies. This case report does not encourage clinicians to use external electrical stimulation as a method of treatment for patients with spondylolysis.

Fellander-Tsai and Micheli ${ }^{22}$ presented a report of two cases, a 16 year old female volleyball player and a 16 year old male soccer player who had spondylolysis at L5. In both cases, the initial assessment showed that lumbar extension reproduced low back pain and that the patients had tight hamstrings and were free of radicular symptoms. Oblique radiographs confirmed the diagnosis, and CT scans showed bilateral pars fractures at L5. The treatment started, for both patients, with a Boston brace worn 23 hours a day and an anti-lordotic exercise programme. In the first evaluation after four months of this treatment, the patients were almost pain free, and both CT scans showed healing on the right side but a gap on the left side. In the second evaluation after 10 months of treatment, the patients reported pain in the lumbar area, and both CT scans showed complete healing on the right side and a persistent gap on the left side. After 14 months of treatment, the patients were in pain and unable to continue normal activities, and both CT scans showed a persistent gap on the left side. At that time, treatment with an external Orthologic 1000 bone growth stimulator (Phoenix, Arizona, USA) was initiated. Treatment was for 30 minutes a day, and the frequency of the signal was 15.3 and $76.6 \mathrm{~Hz}$ with a peak amplitude of $40 \mu \mathrm{T}$. The antilordotic exercise programme was stopped, and the patients stopped wearing the brace. After four months of this treatment, the patients were pain free, and both CT scans showed partial healing on the left side. After one year of treatment, the patients were pain free, and both CT scans showed complete healing of both pars interarticularis defects.

Background information about the subjects' health and how the conditions were diagnosed was presented well. In every assessment, the pain was reproduced by extension, but no outcome measure (visual analogue scale or pain questionnaire) was used to evaluate the level of pain nor was there mention of how the tightness of the hamstrings was measured. The progressive osseous healing was well presented by the use of CT scans. The patients were not advised to restrict their activities. However, the authors did not mention if the patients were informed that restriction of activities helps in the healing of spondylolysis. In addition, they did not report whether the procedure was explained to the patients or if their parents gave permission for their participation. Furthermore, they did not justify why they chose specific dates for reassessment and they did not give any details about the anti-lordotic exercise programme (sets, repetitions, daily or every other day). On the other hand, they did mention what kind of external bone growth stimulator was used, the frequency, and for how long the patients used it, but not what time of the day patients were instructed to use it. However, the description was appropriate and comprehensible. Moreover, they explained why they decided to use external electrical stimulation instead of the antilordotic exercise programme and bracing. In the discussion section, the authors concluded that the healing on the left side occurred as a result of external electrical stimulation, which was used when the initial treatment (bracing and the anti-lordotic exercise programme) failed and the CT scans showed a persistent gap. This article encourages the clinician to use external electrical stimulation as a method of treatment for patients with spondylolysis, but the size of sample is small, consisting of just two patients.

\section{DISCUSSION}

A wide range of conservative interventions has been used for the treatment of spondylolysis. Complete bony healing of the lesion is the ideal goal of treatment. Many authors propose activity restriction. ${ }^{16}{ }^{17}$ Others suggest anti-lordotic bracing ${ }^{18}$ or bracing that maintains lumbar lordosis. ${ }^{8}$ Another important conservative measure advocated is exercise. Abdominal strengthening, hamstring stretching, pelvic tilts, ${ }^{8} 19$ and specific stabilising exercises of the muscles that surround the spine and are considered to provide dynamic stability and fine control to the lumbar spine are some of the exercises often prescribed.$^{20}$ The last conservative intervention that has been used in the treatment of spondylolysis is external electrical stimulation. This treatment is "new" and not mentioned in books. This critical literature review concentrates on two studies that evaluated the efficacy of external 
electrical stimulation in the management of patients with a diagnosis of spondylolysis.

External electrical stimulation was used in adolescent athletes, but in a different ways in the two articles. Pettine et $a l^{21}$ used it from the beginning of the treatment in combination with bracing and restriction of activities, whereas Fellander-Tsai and Micheli ${ }^{22}$ used it when the traditional methods (bracing and anti-lordotic exercise programme) were performed without success. The report of Pettine et al had many flaws in the methods section, whereas that of Fellander-Tsai and Micheli was more detailed and easier to replicate. Pettine et al did not explain whether the pars interarticularis defects healed as a result of the bracing, restriction of activities, or external electrical stimulation, because the patient's pars fractures may have healed with restriction of activities or bracing without electrical stimulation. Studies have shown that treatment for spondylolysis should include some type of immobilisation or activity restriction. On the other hand, Fellander-Tsai and Micheli showed that the pars defects healed as a result of the external electrical stimulation, as the patients had not been advised to restrict their activities. This finding shows that it can be used successfully even if patients have not been advised to restrict their activities.

Electrical stimulation has been used to heal fractures in all areas of the body. The treatment of stress fracture of the lower extremity (navicular and fifth metatarsal) with transcutaneous electrical stimulation has been reported with excellent results. ${ }^{23}$ Mooney $^{24}$ has reported the efficacy of external electrical stimulation as a supplement to bone grafting in spinal fusion. The rate of lumbar spinal fusion improved significantly compared with a control group. Rettig et $a l^{25}$ recommended a trial of external stimulation before considering surgery in patients with anterolateral "black line" stress fracture of the tibia. Spondylolysis is a stress or fatigue fracture of the pars interarticularis, and the use of external electrical stimulation to heal this condition seems to be logical.

Although Fellander-Tsai and Micheli showed that external electrical stimulation can decrease pain and heal pars defects, we cannot conclude whether it is more effective than other conservative interventions or whether it can be used for the treatment of this condition, because the size of sample is too small and the results cannot be generalised to the rest of the population. The other conservative methods have been used in experimental studies with a sufficiently large and thus representative sample, so their results can be generalised to the rest of the population. Further studies with more patients and more detailed procedures are needed to establish what the exact role of external electrical stimulation should be in the management of patients with spondylolysis.

\section{REFERENCES}

1 Smith J, Hu S. Management of spondylolysis and spondylolisthesis in the pediatric and adolescent population. Disorders of the Pediatric and Adolescent Spine 1999;30:487-99.

2 Dubousset J. Treatment of spondylolysis and spondylolisthesis in children and adolescents. Clin Orthop 1997;24:1342-55.

3 Morita T, Ikata T, Katoh S, et al. Lumbar spondylolysis in children and adolescents. J Bone Surg [Br] 1995;77:620-5.

4 O'Neil D, Micheli L. Postoperative radiographic evidence for fatigue fracture as the etiology of spondylolysis. Spine 1989;1:1342-55.

5 Ikata T, Miyake R, Katoh S, et al. Pathogenesis of sports related spondylolisthesis in adolescents. Am J Sports Med 1996;24:94-8.

6 Micheli L, Wood R. Back pain in young athletes. Arch Pediatr Adolesc Med 1995; 149:15-18.

7 Turner P, Green J, Galasco S. Back pain in childhood. Spine 1989;1:812-14.

8 Blanda J, Bethem D, Moats W. Defects of pars interarticularis in athletes: a protocol for nonoperative treatment. J Spinal Disord 1993;6:406-11.

9 Garry J, McShane J. Lumbar spondylolysis in adolescent athletes. Spine 1988;47:145-9.

10 Green P, Alley C, Adams A. Spondylolysis: bending of the inferior processes of lumbar vertebrae during simulated spinal movements. Spine 1994; 19:2683-91.

11 Simper L. Spondylolysis in Eskimo skeletons. Acta Orthop Scand 1986;57:78-80.

12 Soren A, Waugh T. Spondylolisthesis and related disorders: a correlative study of 105 patients. Clin Orthop 1985;143:171-7.

13 Jackson W, Wiltse L, Dingeman $D$, et al. Stress reaction involving the pars interarticularis in young athletes. Am J Sports Med 1981;9:304-12.

14 Hensinger R. Spondylolysis and spondylolisthesis in children and adolescents. $J$ Bone Joint Surg [Am] 1989;71:1098-107.

15 Bell F, Ehrlich G, Zaleske J. Brace treatment for symptomatic spondylolisthesis. Clin Orthop 1988;236:192-8.

16 Pezzulo D. Spondylolysis and spondylolisthesis in athletes. Athletic Therapy Today 1999;4:36-40.

17 Johnson M. Low back pain in sports. Phys Sportsmed 1993;21:53-9.

18 Steiner M, Micheli L. Treatment of symptomatic spondylolysis and spondylolisthesis with the modified Boston brace. Spine 1985;10:937-43.

19 Daniel J, Polly D, Van Dam B. A study of the efficacy of nonoperative treatment of presumed traumatic spondylolysis in a young patient population. Mil Med 1995; 160:553-5

20 O'Sullivan D, Twomey T, Allison G. Evaluation of specific stabilising exercise in the treatment of chronic low back pain with radiologic diagnosis of spondylolysis or spondylolisthesis. Spine 1997;22:2959-67.

21 Pettine K, Salib R, Walker S. External electrical stimulation and bracing for treatment of spondylolysis. A case report. Spine 1993;18:436-9.

22 Fellander-Tsai L, Micheli L. Treatment of spondylolysis with external electrical stimulation: a report of two cases. Clin J Sports Med 1998;8:232-4.

23 Benazzo F, Mosconi M, Beccarisi G, et al. Use of capacitive coupled electric fields in stress fractures in athletes. Clin Orthop 1995; 31:145-9.

24 Mooney V. A randomized double blind prospective study of the efficacy of pulsed electromagnetic fields for interbody lumbar fusions. Spine 1990;15:708-12.

25 Retting C, Shelbourne D, McCarroll R, et al. The natural history and treatment of delayed union stress fractures of the anterior cortex of the tibia. Am J Sports Med 1988; 10:250-5. 